\title{
Effect of Cervical Length Recovery after Laser Surgery for Twin-Twin Transfusion Syndrome
}

\author{
Matthew Finneran, MD ${ }^{1} \quad$ Lorene Temming, $\mathrm{MD}^{2} \quad$ Megan Templin, MS 3 Courtney Stephenson, DO ${ }^{1}$ \\ ${ }^{1}$ Department of Obstetrics and Gynecology, Charlotte Fetal Care \\ Center, Charlotte, North Carolina \\ 2 Department of Obstetrics and Gynecology, Washington University, \\ St. Louis, Missouri \\ ${ }^{3}$ Dickson Advanced Analytics, Carolinas Medical Center, Charlotte, \\ Address for correspondence Matthew Finneran, MD, Department of \\ Obstetrics and Gynecology, Carolinas Medical Center, 1000 Blythe \\ Blvd., Charlotte, NC \\ (e-mail: matthew.finneran@carolinashealthcare.org).
} North Carolina

Am J Perinatol Rep 2016;6:e137-e141.

\begin{abstract}
Keywords

- cervical length

- twin-twin transfusion syndrome

- laser

- polyhydramnios

Objective To evaluate the impact of polyhydramnios on preoperative cervical length and whether cervical length recovery after amnioreduction during selective fetoscopic laser photocoagulation (SFLP) is associated with a greater gestational age at delivery in pregnancies complicated by twin-twin transfusion syndrome (TTTS).

Methods Retrospective study of 50 pregnancies complicated by TTTS treated with SFLP between March 2010 and July 2014 at a single center. Preoperative maximum vertical pocket (MVP) was measured along with pre- and postoperative cervical length. $A$ cervical length difference was calculated, with a difference of $\pm 3 \mathrm{~mm}$ considered no change.

Results Only 12 (34\%) patients showed an increased cervical length after amnioreduction during laser surgery. There was no statistical difference between either negative or positive change in cervical length groups and mean gestational age at delivery $(p=0.82)$. There also was no correlation between preoperative MVP and preoperative cervical length $(p=0.36)$ or gestational age at delivery $(p=0.77)$. However, there was a statistically shorter mean preoperative cervical length in patients who delivered $<32$ weeks of gestation ( $3.62 \pm 0.66$ vs. $4.20 \pm 0.85 \mathrm{~cm} ; p=0.03$ ).

Conclusion Severity of polyhydramnios does not correlate with preoperative cervical length, and variability of the cervix postoperatively does not appear to affect gestational age at delivery.
\end{abstract}

Laser ablation of the placental vascular equator has emerged as the standard of care in moderate-severe twin-twin transfusion syndrome (TTTS). ${ }^{1,2}$ Although laser surgery has had a significant impact on survival, adverse neonatal outcomes remain common secondary to a high rate of prematurity. ${ }^{3,4}$

In several studies, a shortened cervical length has been identified as an independent risk factor for preterm birth in pregnancies treated with laser surgery. However, there has been significant heterogeneity to the concluded preoperative cervical length cut-off values that predispose toward increased risk of preterm birth after laser surgery. ${ }^{3,5-7}$

received

June 1, 2015

accepted after revision

January 19, 2016
DOI http://dx.doi.org/

10.1055/s-0036-1579654. ISSN 2157-6998.
The etiology of cervical shortening in TTTS is multifactorial, but the presence of severe polyhydramnios leading to an increase in intrauterine pressure has been identified as a contributing factor. ${ }^{8}$ Given that polyhydramnios may have a negative impact on cervical length, amnioreduction during laser surgery may increase cervical length postoperatively. Cervical length recovery after amnioreduction may identify a patient population with better outcomes with respect to gestational age at birth.

The objective of this study is to evaluate the impact of polyhydramnios on preoperative cervical length and whether
Copyright $\odot 2016$ by Thieme Medical Publishers, Inc., 333 Seventh Avenue, New York, NY 10001, USA. Tel: +1(212) 584-4662.
License terms

(요 (1) $\Theta$ 
cervical length recovery after amnioreduction during selective fetoscopic laser photocoagulation (SFLP) is associated with a greater gestational age at delivery in pregnancies complicated by severe TTTS.

\section{Materials and Methods}

\section{Study Design}

This was a retrospective study of all patients with confirmed monochorionic-diamniotic twin gestation complicated by TTTS who underwent SFLP at the Charlotte Fetal Care Center from March 2010 to July 2014. All patients signed a consent form for release of their records prior to the procedure, and there is a standing institutional review board (IRB) approval for research regarding data in the fetal care database (Carolinas Medical Center IRB \# 08-10-09E). As part of a standard evaluation during consultation, patients undergo a comprehensive ultrasound during which the cervical length and maximum vertical pocket (MVP) are measured. At our center, there are only two ultrasonographers involved with this assessment. Patients are staged for TTTS with both the Quintero and Cincinnati systems. All patients who met criteria and desired surgical management underwent the procedure within 24 hours, but not more than 72 hours. The procedure is performed under regional anesthesia with a single port. The standard surgical technique at our institution employs placental mapping and selective laser ablation of identified unpaired vascular anastomoses. After the laser portion is complete, an amnioreduction is performed to reduce the MVP to approximately 7 to $8 \mathrm{~cm}$. Immediately after the procedure, the cervical length is again measured. Patients are followed at our center until improvement or resolution of TTTS is observed, after which they return to their referring providers for further management.

Exclusion criteria included presentation at $>26$ weeks of gestation, prior preterm delivery at $<34$ weeks of gestation, higher order multiple gestations, and known fetal aneuploidy or anomalies. Maternal demographics were obtained from the medical record, including age, parity, prior preterm deliveries, race, major medical illnesses, and Quintero/Cincinnati stage at time of scheduling of procedure. The initial MVP at time of consultation, preoperative cervical length, and postoperative cervical length were recorded. The difference between the preoperative and postoperative cervical length was calculated.

In post hoc analysis, patients were separated into three groups based upon whether they gained, lost, or had no change in their cervical length postprocedure. No change was defined as $\pm 3 \mathrm{~mm}$, which has been shown in previous studies to be the interobserver variability of transvaginal cervical length measurements. ${ }^{9,10}$

When analyzing variables with respect to latency from procedure or gestational age at delivery, those patients with deliveries secondary to maternal or fetal indications prior to 34 weeks were excluded unless preceded by spontaneous labor. Spontaneous labor was defined as either a documentation of preceding preterm premature rupture of membranes (PPROM) or spontaneous contractions with cervical change. This information was obtained from both our institutional and outside hospital records.

\section{Statistical Analysis}

Descriptive statistics, including means, medians, and standard deviations, or counts and percentages, were calculated. For normally distributed data measured on an interval scale, Student $t$-test and analysis of variance were used. If the data were ordinal or not normally distributed, the Wilcoxon ranksum test or the Kruskal-Wallis test was employed. For nominal data, the chi-square test or Fisher exact test was performed. To test for linear relationships among variables, Pearson and Spearman correlation coefficients were calculated. SAS Enterprise Guide, (SAS Institute Inc., Cary, NC) version 5.1 , was used for all analyses. A two-tailed $p$-value of $<0.05$ was considered statistically significant.

\section{Results}

During the study period, 54 women underwent SFLP at our institution. There were four patients with missing postoperative cervical length data and were excluded from the analysis. Of the remaining 50 patients, 15 (30\%) experienced nonspontaneous preterm birth at $<34$ weeks of gestation for fetal or maternal indications. The demographics of the study population are depicted in -Table $\mathbf{1}$.

In 9 (26\%) patients, the cervical length was longer, in 12 (34\%) patients the cervical length was shorter, and in $14(40 \%)$ patients the cervix did not change after the procedure. The average cervical length change was -0.81 and $+0.69 \mathrm{~cm}$ for a gain and loss of length, respectively. The mean postoperative cervical lengths were $3.42,4.0$, and $4.18 \mathrm{~cm}$ in the negative difference, no change, and positive difference groups, respectively. However, these differences were not statistically significant. There was no statistical significance in whether there was any change in the cervical length postoperatively with respect to latency from procedure and gestational age at delivery (-Table 2 ).

There were five (14\%) patients among the study population who had a cerclage placed prior to the procedure. However, these patients were evenly distributed between the gained and lost cervical length groups. The average cervical length change of all patients with cerclage was $-0.28 \mathrm{~mm}$, which is within the interobserver variability of cervical length measurements. There was also no statistical difference in the mean gestational age at delivery from the rest of the cohort.

There was no statistically significant correlation between preoperative and postoperative cervical length and gestational age at delivery. However, those who delivered at less than 32 weeks had a shorter preoperative cervical length than those who delivered after this gestation (3.62 vs. $4.20 \mathrm{~cm}$; $p=0.032$; - Table 3).

The initial MVP at consultation was analyzed in the entire cohort $(n=50)$ with respect to preoperative cervical length. There was no correlation found between these two variables $(r=-0.13 ; p=0.36 ;-$ Fig. 1$)$. When excluding nonspontaneous preterm birth $<34$ weeks of gestation, there was no correlation of MVP with either latency from procedure $(r=-0.06 ; p=0.74)$ or gestational age at delivery $(r=0.05 ; p=0.76)$. 
Table 1 Population characteristics

\begin{tabular}{|c|c|c|c|}
\hline & Indicated delivery $<34$ wk $(n=15)$ & Spontaneous birth $(n=35)$ & $p$ \\
\hline Multiparous, $\%$ & $11(73.3)$ & $24(68.6)$ & 0.74 \\
\hline Caucasian, \% & $11(73.3)$ & $23(65.7)$ & 0.75 \\
\hline Anterior placenta, \% & $10(66.7)$ & $12(34.3)$ & 0.04 \\
\hline Maternal age (mean \pm SD), y & $25.6 \pm 6.19$ & $29.9 \pm 5.4$ & 0.02 \\
\hline GA at surgery (mean $\pm S D$ ), wk & $21.0 \pm 2.8$ & $21.3 \pm 2.3$ & 0.67 \\
\hline $\mathrm{GA}$ at delivery (mean $\pm \mathrm{SD}$ ), wk & $28.4 \pm 4.23$ & $31.5 \pm 3.97$ & 0.02 \\
\hline \multicolumn{4}{|l|}{ Cincinnati stage, \% } \\
\hline 3 & $1(6.7)$ & $2(5.7)$ & \multirow[t]{5}{*}{0.4} \\
\hline $3 A$ & $2(13.3)$ & $5(14.3)$ & \\
\hline 3B & $3(20.0)$ & $15(42.9)$ & \\
\hline $3 C$ & $9(60.0)$ & $12(34.3)$ & \\
\hline 4 & $0(0)$ & $1(2.9)$ & \\
\hline \multicolumn{4}{|l|}{ Quintero stage, \% } \\
\hline 1 & $0(0)$ & $2(5.7)$ & \multirow[t]{4}{*}{0.71} \\
\hline 2 & $0(0)$ & $3(8.6)$ & \\
\hline 3 & $15(100)$ & 29 (82.9) & \\
\hline 4 & $0(0)$ & $1(2.9)$ & \\
\hline Cerclage, \% & $2(13.3 .0)$ & $5(14.3)$ & 0.93 \\
\hline
\end{tabular}

Abbreviation: GA, gestational age.

Table 2 Change in cervical length postoperatively

\begin{tabular}{|l|l|l|l|l|}
\hline & Negative difference & No change & Positive difference & $p$ \\
\hline$N$ & 12 & 14 & 9 & $21.43 \pm 1.81$ \\
\hline GA at surgery (mean \pm SD), wk & $20.33 \pm 2.08$ & $22.00 \pm 2.64$ & $3.49 \pm 0.41$ & 0.1861 \\
\hline Pre-op CL (mean \pm SD), cm & $4.23 \pm 1.04$ & $3.97 \pm 0.7$ & $4.18 \pm 0.69$ & 0.1129 \\
\hline Post-op CL (mean \pm SD), cm & $3.42 \pm 0.97$ & $4 \pm 0.78$ & $0.69 \pm 0.44$ & 0.0931 \\
\hline CL difference (mean \pm SD), cm & $-0.81 \pm 0.52$ & $0.03 \pm 0.21$ & $66 \pm 27.21$ & $<0.0001$ \\
\hline Latency (mean \pm SD), d & $78.17 \pm 36.24$ & $69.79 \pm 31.51$ & $30.86 \pm 3.06$ & 0.6694 \\
\hline GA at birth (mean \pm SD), wk & $31.5 \pm 5$ & $31.97 \pm 3.7$ & $12.82 \pm 2.33$ & 0.8152 \\
\hline MVP (mean \pm SD), cm & $11.04 \pm 1.97$ & $12.12 \pm 2.64$ & 0.2272 \\
\hline
\end{tabular}

Abbreviations: $\mathrm{CL}$, cervical length; GA, gestational age; MVP, maximum vertical pocket.

Table 3 Variables compared with gestational ages at delivery

\begin{tabular}{|l|l|l|l|}
\hline & $<32$ wk $(\boldsymbol{n}=16)$ & $>32$ wk $(\boldsymbol{n}=\mathbf{1 9})$ & $\boldsymbol{p}$ \\
\hline GA at surgery (mean \pm SD), wk & $21.61 \pm 2.09$ & $21.01 \pm 2.52$ & 0.4539 \\
\hline Pre-op CL (mean \pm SD), cm & $3.62 \pm 0.66$ & $4.20 \pm 0.85$ & 0.0320 \\
\hline Post-op CL (mean \pm SD), cm & $3.6 \pm 0.76$ & $4.06 \pm 0.92$ & 0.1231 \\
\hline CL difference (mean \pm SD), mm & $-0.02 \pm 0.61$ & $-0.15 \pm 0.79$ & 0.6030 \\
\hline MVP (mean \pm SD), cm & $11.92 \pm 2.42$ & $11.93 \pm 2.42$ & 0.9936 \\
\hline
\end{tabular}

Abbreviations: $\mathrm{CL}$, cervical length; $\mathrm{GA}$, gestational age; MVP, maximum vertical pocket. 


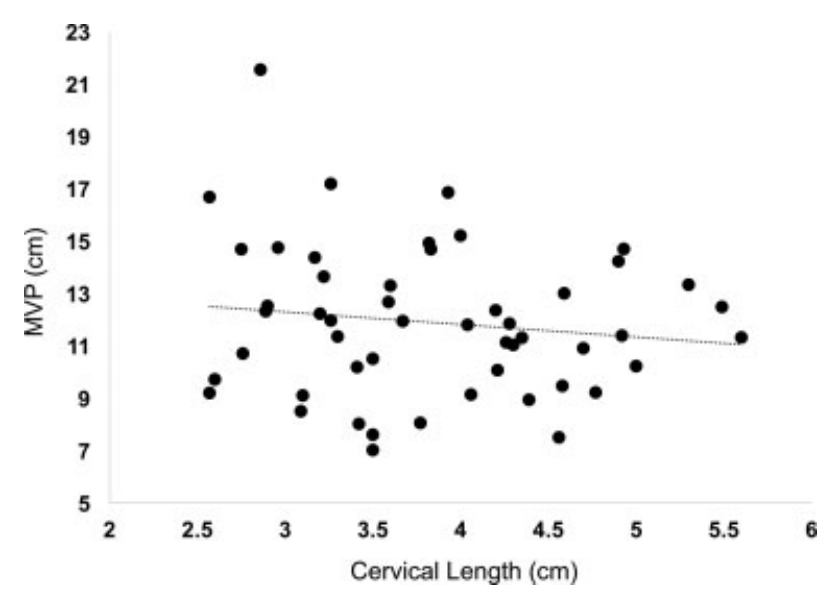

Fig. 1 MVP versus preoperative cervical length $(r=-0.13 ; p=0.36)$.

\section{Discussion}

Our study shows that cervical length does not correlate to the severity of polyhydramnios prior to laser therapy for TTTS and suggests that change in cervical length postoperatively has no prognostic value in prediction of latency from procedure or gestational age at birth.

There does not appear to be any prognostic value in whether there was cervical length change after amnioreduction during laser therapy for TTTS. However, there was no significant difference in the postoperative cervical lengths regardless of degree of change. This result would be expected given the lack of correlation between preoperative MVP and cervical length. If severity of polyhydramnios preoperatively does not affect cervical length, it is logical that amnioreduction would not have an impact on cervical length either. It is important to note that the negative cervical length group had a higher average preoperative measurement $(4.34 \mathrm{~cm})$ and the positive cervical length group had a lower average preoperative measurement $(3.51 \mathrm{~cm})$. This would suggest that the shorter the cervical length preoperatively, the more potential there is for cervical length recovery. However, the change in cervical length, regardless of whether shortened or lengthened, does not appear to be clinically significant.

In our study, there were significantly less patients with cervical length change postoperatively as compared with prior research. Cobo et al also analyzed this variable with $43.9 \%$ (73/166) of patients having an increase in cervical length postprocedure. ${ }^{6}$ However, measurements were performed 24 hours postoperatively and it is unclear if measurements were controlled for interobserver variability, which may explain the differences in our results.

Previous literature has suggested that polyhydramnios in singleton gestations is associated with preterm birth. ${ }^{11}$ The unique pathophysiology of polyhydramnios in TTTS may explain why cerclage has been utilized in TTTS pregnancies with some positive effect ${ }^{4}$ but has proved detrimental in uncomplicated twin gestations. ${ }^{12}$ However, this initially encouraging result has been called into question by a recent retrospective review. ${ }^{13}$ In our study, MVP preoperatively did not correlate with either latency from procedure to delivery or gestational age at delivery. This may be explained by the effectiveness of laser surgery in resolving the fluid derangements in TTTS and eliminating recurrent polyhydramnios as a contributing factor to uterine distension and subsequent preterm labor.

In our study, neither preoperative nor postoperative cervical length had a statistically significant correlation with either latency from procedure or gestational age at birth. This is discordant with prior research in this area. $3,5,6,14$ However, our results may be secondary to sample size and the multifactorial nature of preterm birth, especially in patients treated with laser therapy. Our study may not be powered to detect this difference as other variables, such as the high rate of PPROM in laser patients, may have a greater impact on gestational age at delivery than cervical length. Also, the average pre- and postoperative cervical length was above $3 \mathrm{~cm}$ in all groups and no patient had a cervical length $<2.5 \mathrm{~cm}$ (except prior to cerclage). It is likely that our cohort does not represent a high-risk group for preterm delivery with respect to cervical length. Despite these findings, there was a shorter average preoperative cervical length in those patients who delivered $<32$ weeks. The fact that there was no correlation between cervical length and gestational age at delivery in the entire cohort, but a statistically shorter cervical length in patients delivering $<32$ weeks may be secondary to our inclusion criteria as we included patients with nonspontaneous birth who delivered $>34$ weeks. Given that many centers advocate for delivery between 34 and 36 weeks, these patients may have affected these results.

One of the strengths of this study is its single-center design with only one surgeon and two ultrasonographers involved in the care of our TTTS patients, which mitigates the impact of surgical technique and ultrasound measurement variability. We excluded patients with iatrogenic delivery $<34$ weeks to prevent confounding results, but this significantly decreased our sample size. Also, this study is retrospective in nature with inherent potential for bias. There were four patients who could not be included in the analysis, as the cervical length was not documented in the ultrasound report. Given that we are a referral center, referring physicians manage most patients postoperatively once improvement or resolution of TTTS is evident, which can introduce bias into the management and timing of delivery.

\section{Conclusion}

Cervical length remains an important prognostic factor in the prediction of preterm birth in patients treated with laser in TTTS. However, severity of polyhydramnios does not correlate with preoperative cervical length, and variability of the cervix postoperatively does not appear to affect gestational age at delivery.

\section{Funding}

No funding was received for this study.

\section{Conflict of Interest}

The authors have no conflicts of interest to disclose. 


\section{Note}

This study was presented at the International Fetal Medicine and Surgery Society Annual Meeting on September 8, 2014, in Chatham, MA.

\section{References}

1 Slaghekke F, Lopriore E, Lewi L, et al. Fetoscopic laser coagulation of the vascular equator versus selective coagulation for twin-to-twin transfusion syndrome: an open-label randomised controlled trial. Lancet 2014;383(9935):2144-2151

2 Senat M-V, Deprest J, Boulvain M, Paupe A, Winer N, Ville Y. Endoscopic laser surgery versus serial amnioreduction for severe twin-to-twin transfusion syndrome. N Engl J Med 2004;351(2): 136-144

3 Robyr R, Boulvain M, Lewi L, et al. Cervical length as a prognostic factor for preterm delivery in twin-to-twin transfusion syndrome treated by fetoscopic laser coagulation of chorionic plate anastomoses. Ultrasound Obstet Gynecol 2005;25(1):37-41

4 Salomon LJ, Nasr B, Nizard J, et al. Emergency cerclage in cases of twin-to-twin transfusion syndrome with a short cervix at the time of surgery and relationship to perinatal outcome. Prenat Diagn 2008;28(13):1256-1261

5 Papanna R, Mann LK, Baschat AA, et al. Cervical length in prediction of preterm birth after laser surgery for twin-twin transfusion syndrome. Ultrasound Obstet Gynecol 2015;45(2):175-182

6 Cobo T, Palacio M, Eixarch E, et al. Clinical and biochemical predictors of very preterm birth in twin-to-twin transfusion syndrome treated by fetoscopy. Am J Obstet Gynecol 2011; 204(1):58.e1-58.e5
7 Chavira ER, Khan A, Korst LM, Miller D, Goodwin TM, Chmait RH. Are patients with twin-twin transfusion syndrome and a very short cervix candidates for laser surgery? J Ultrasound Med 2009; 28(5):633-639

8 Engineer N, O'Donoghue K, Wimalasundera RC, Fisk NM. The effect of polyhydramnios on cervical length in twins: a controlled intervention study in complicated monochorionic pregnancies. PLoS ONE 2008;3(12):e3834

9 Valentin L, Bergelin I. Intra- and interobserver reproducibility of ultrasound measurements of cervical length and width in the second and third trimesters of pregnancy. Ultrasound Obstet Gynecol 2002;20(3):256-262

10 Stein W, Hellmeyer L, Schmidt S, Tekesin I. Intraobserver and interobserver reliability of transvaginal cervical length measurements and quantitative ultrasound tissue characterization of the cervix in the second and third trimester of pregnancy. Ultraschall Med 2011;32(Suppl 2):E169-E174

11 Pri-Paz S, Khalek N, Fuchs KM, Simpson LL. Maximal amniotic fluid index as a prognostic factor in pregnancies complicated by polyhydramnios. Ultrasound Obstet Gynecol 2012;39(6):648-653

12 Berghella V, Odibo AO, To MS, Rust OA, Althuisius SM. Cerclage for short cervix on ultrasonography: meta-analysis of trials using individual patient-level data. Obstet Gynecol 2005;106(1): 181-189

13 Papanna R, Habli M, Baschat AA, et al. Cerclage for cervical shortening at fetoscopic laser photocoagulation in twin-twin transfusion syndrome. Am J Obstet Gynecol 2012;206(5):425. e1-425.e7

14 Chmait RH, Korst LM, Llanes A, Mullin P, Lee RH, Ouzounian JG. Perioperative characteristics associated with preterm birth in twin-twin transfusion syndrome treated by laser surgery. Am J Obstet Gynecol 2013;209(3):264.e1-264.e8 